\title{
RESIDENT'S CORNER
}

\section{The Evaluation of an Osteoporosis Clinic in a Community Hospital Setting: a Retrospective Chart Review and Telephone Survey}

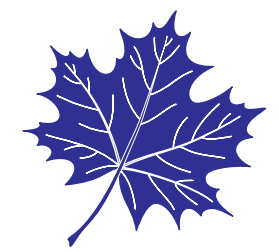

\author{
Timothy S.H. Kwok, MD ${ }^{1}$, Natasha Gakhal, MD, MSc, FRCPC ${ }^{2,3}$, Thanu N. Ruban, MD, FRCPC ${ }^{3,4}$ \\ ${ }^{1}$ Department of Medicine, Faculty of Medicine, University of Toronto, Toronto, ON; ${ }^{2}$ Women's College Hospital, Toronto, \\ ON; ${ }^{3}$ Division of Rheumatology, Department of Medicine, Faculty of Medicine, University of Toronto, Toronto, ON; \\ ${ }^{4}$ Markham Stouffville Hospital, Markham, ON, Canada
}

DOI:https://doi.org/10.5770/cgj.22.359

\begin{abstract}
\section{Background}

Patients who have suffered fragility fractures are at an increased risk for subsequent fractures. The Osteoporosis (OP) Clinic at Markham Stouffville Hospital (MSH) was set up in July 2015 to screen, diagnose, and treat patients with fragility fractures. The goal of this study was to identify differences in OP screening and treatment initiation between patients seen in the OP clinic versus usual care.
\end{abstract}

\section{Methods}

A retrospective cohort study and telephone interview was conducted on 40 patients who had sustained a hip fragility fracture between September 2015 and July 2016. 20 of those patients were referred to the OP clinic, while the remaining patients received usual care.

\section{Results}

At the end of the intervention, $16 / 20$ patients in the OP clinic group were appropriately placed on a bisphosphonate/ RANKL inhibitor versus only $6 / 20$ patients in the usual care group $(p<.01)$.

\section{Conclusions}

A significant care gap exists in secondary fracture prevention between the osteoporosis clinic and usual care groups. Better screening and subsequent intervention are needed for patients with fragility fractures. This study highlights the efficacy of an outpatient OP clinic in a community hospital setting.

Key words: osteoporosis, quality improvement, community medicine

\section{INTRODUCTION}

Patients who have sustained previous fragility fractures are at an increased risk of future secondary fractures. ${ }^{(1)}$ To prevent subsequent fractures, the concept of a novel Fracture Liaison Service (FLS) was established in 1999 in Scotland to enhance the treatment of osteoporosis. ${ }^{(2)}$ The FLS targets osteoporosis risk factors, makes use of diagnostic imaging, and offers appropriate treatments. $^{(3)}$ To date, FLS programs have been implemented worldwide, and its success in secondary prevention of fragility fractures has been widely documented. ${ }^{(4)}$

The Osteoporosis (OP) clinic at Markham Stouffville Hospital (MSH) was set up in July 2015 to screen, diagnose, and treat patients with fragility fractures. The referral process consists of a central nurse coordinator who reviews all consultations seen at the MSH fracture clinic. Patients who, via clinical history, fit the criteria for a fragility fracture are subsequently referred to the OP clinic. The target population consists of patients who have previously sustained a fracture and have been treated at the MSH fracture clinic, with the main goal of preventing future fragility fractures. One point of difference between the MSH OP clinic and a traditional FLS is the solely outpatient focus of the OP clinic. The OP clinic involves a multidisciplinary team of health-care practitioners including nurses, rheumatologists, endocrinologists, and general internists. The goal of this study was to identify differences in OP screening and treatment initiation between patients seen at the MSH OP clinic versus usual care, to determine the care gaps in secondary fracture prevention in a community hospital setting.

\section{METHODS}

A retrospective cohort study and telephone interview was conducted on sequentially sampled patients who sustained a hip fragility fracture between September 2015 and July 2016 inclusive at Markham Stouffville Hospital, a 265-bed 
acute care and community hospital with a catchment area of approximately 400,000 people. As this was a pilot quality improvement study, the number of 40 total patients was decided upon by the authors prior to patient recruitment. All 40 patients were seen at a fracture clinic by an orthopaedic surgeon post hip fracture. Of these patients, 20 consecutive patients who were evaluated at the OP clinic through the fracture clinic were placed into the OP clinic group for the purposes of this study. Patients were selected by a central nurse coordinator for referral to the OP clinic based on history of a fragility fracture. Patients with referral forms that documented evidence of sustaining a fragility fracture were subsequently referred to the OP clinic.

Only patients who had sustained a hip fragility fracture were included in this study. A total of 22 patients were screened by the authors to meet the study number of $20 \mathrm{pa}-$ tients in the OP clinic group (Figure 1). The other 20 consecutive patients were seen in the fracture clinic and subsequently followed up with a primary care provider ('the usual care group'). Forty-four patients were screened by the authors to meet the study number of 20 patients in the usual care group (Figure 1). Patients seen at the fracture clinic may or may not have been referred initially to the OP clinic, but nonetheless declined evaluation at the OP clinic. All 40 patients' charts were then thoroughly reviewed to confirm that the patient had sustained a fragility hip fracture (i.e., fall from standing height or less), regardless of inclusion in the OP or usual care groups. Patients with a hip fracture were specifically selected for this chart audit because they are at high risk of increased morbidity and mortality one year post-fracture. ${ }^{(5)}$ Patients with stage IV/V chronic kidney disease, severe dementia, a palliative condition, or those in long-term care facilities were excluded from the study. Student's $t$ test was used for nominal characteristics, while the Pearson's $\chi^{2}$ test was used for categorical variables. Statistics were calculated using IBM SPSS (IBM Corporation, 2017). The study was approved by the Research Ethics Board at Markham Stouffville Hospital.

\section{RESULTS}

The average age of patients included in this study was 80.4 years. During the study period, 112 total patients were seen at the OP clinic. Twenty-two patients' charts were analyzed to fulfill the sequential sample of 20 patients for the OP clinic group, as one was deceased at the time of data collection and the other resided in long-term care. Forty-four patients' charts were analyzed to fulfill the sequential sample of 20 patients in the usual care group due to a variety of reasons (Figure 1). Demographic, clinical, imaging, and pharmacotherapy data were obtained from electronic medical records. The telephone survey was conducted from August to September 2016. Baseline demographic information is noted in Table 1. There was no significant difference in age or gender distribution between the two groups $(p>.5)$. Moreover, risk factors for osteoporosis that were present in the OP clinic group included current or previous glucocorticoid use $-4 / 20(20 \%)$, parental hip fracture $-1 / 20(5 \%)$, current smoking status $-1 / 20$ (5\%), and $>3$ alcoholic drinks per week $-1 / 20$ (5\%). Mean bone mineral density (BMD) T-score of the lowest site by dual-energy X-ray absorptiometry for the OP clinic patients was $-2.28(-0.2--3.9)$ (Table 2). All OP clinic patients had a BMD. The BMD information was not available for patients in the usual care group.

At the end of the intervention, $16 / 20(80 \%)$ patients in the OP clinic group were appropriately placed on a bisphosphonate/RANKL inhibitor, while only $6 / 20(30 \%)$ patients in the usual care group were on a bisphosphonate/RANKL

Osteoporosis Clinic group

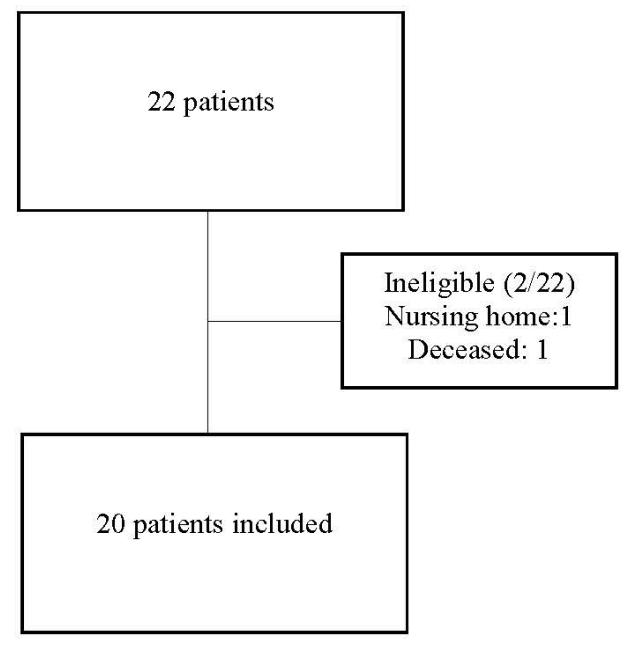

Usual Care group

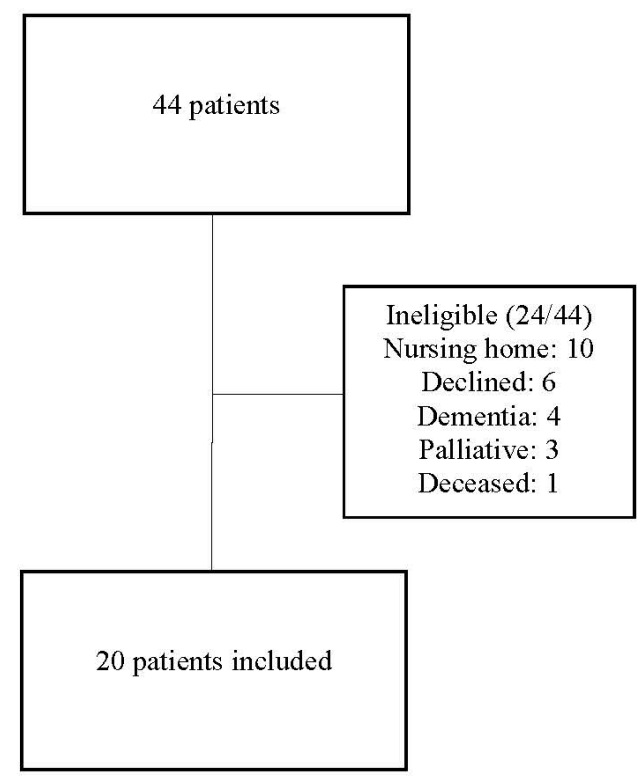

FIGURE 1. Inclusion/exclusion flowchart of the Osteoporosis Clinic and Usual Care groups 
TABLE 1.

Baseline demographic information and bisphosphonate/RANKL inhibitor use for patients in the Osteoporosis Clinic and Usual Care groups

\begin{tabular}{lcc}
\hline & $\begin{array}{c}\text { Osteoporosis Clinic Group } \\
(n=20)\end{array}$ & $\begin{array}{c}\text { Usual Care Group } \\
(n=20)\end{array}$ \\
\hline Age & $79.3(58-97)$ & $81.5(69-93)$ \\
Sex (F:M) & $16: 4$ & $15: 5$ \\
Bisphosphonate/RANKL inhibitor prior to fragility fracture & 4 & 5 \\
\hline
\end{tabular}

TABLE 2

Clinical parameters and telephone survey responses for patients in the Osteoporosis Clinic group ( $n=20)$

\begin{tabular}{|c|c|c|}
\hline \multirow[t]{6}{*}{ Osteoporosis Risk Factors } & $\begin{array}{c}\text { Menopausal } \\
\text { Average number of previous fragility fractures }\end{array}$ & $\begin{array}{c}16 \\
1.5(1-2)\end{array}$ \\
\hline & Patients with parental fractured hip & 1 \\
\hline & Currently smoking & 1 \\
\hline & Glucocorticoid use & 2 \\
\hline & Rheumatoid arthritis & 0 \\
\hline & Alcohol use ( $>3$ drinks/wk) & 1 \\
\hline \multirow[t]{8}{*}{ Osteoporosis Clinic Intervention Information } & Wait-time to see Osteoporosis Clinic physician (days) & $63.7(8-20)$ \\
\hline & Bone mineral density (BMD) testing post-fragility fracture & 20 \\
\hline & Average $\mathrm{BMD}^{\mathrm{b}}$ & $-2.28(-0.2--3.9)$ \\
\hline & Bisphosphonate/RANKL inhibitor prescribed & 12 \\
\hline & Bisphosphonate & $9 / 12$ \\
\hline & RANKL inhibitor & $3 / 12$ \\
\hline & Osteoporosis pharmacotherapy post-fragility fracture & 16 \\
\hline & Medication adherence 3-months post-treatment initiation & $12 / 16$ \\
\hline
\end{tabular}

ancluding current fragility fracture.

${ }^{\mathrm{b}}$ Lowest T-score at all sites.

inhibitor $(p<.01)$ (Tables 2 and 3). A bisphosphonate/ RANKL inhibitor was prescribed to 60 per cent $(12 / 20)$ of the OP clinic patients who were deemed eligible. The other four patients in the OP clinic group were already on OP treatment prior to their fracture. Self-reported compliance with the prescribed treatment was $12 / 16(75 \%)$ at three months after treatment initiation (Table 2).

In the usual care group, 10/20 (50\%) patients had a BMD test arranged by their health-care provider after their fracture, while all patients in the OP clinic group had a BMD post-fracture (Tables 2 and 3). Five of twenty (25\%) patients were taking OP treatment prior to their fracture in the usual care group. Following usual care, one additional patient was initiated on OP treatment. Despite usual care by primary care providers, five patients were referred to the OP clinic for assessment. However, 8/15 (53.3\%) patients in the usual care group were not referred to the OP clinic despite meeting referral criteria (Table 3).

\section{DISCUSSION}

Canadian guidelines recommend the initiation or continuation of pharmacotherapy post-hip fragility fracture.
(6) However, only 6/20 patients were on OP treatment postfracture in the usual care group, compared with 16/20 in the OP clinic group. Hence, a significant care gap exists in secondary fracture prevention between the two groups. The difference between the lower study participant rate for the usual care group is due to fewer patients meeting our inclusion criteria - a greater number of total patients in the usual care group had dementia, were palliative, in long-term care, or declined participation (Figure 1).

All patients who have suffered a fragility fracture should undergo a comprehensive clinical assessment for osteoporosis. Although 19/20 patients in the usual care group visited their family physicians for a follow-up post-fracture, only 10 patients underwent BMD testing. From a resource allocation perspective, patients should follow-up with their primary care providers for osteoporosis assessment. However, this may not be completed in a timely manner or completed at all, for a number of reasons. ${ }^{(7)}$ Barriers to primary care providers' post-fracture OP assessment may include timely access, knowledge gap, and lack of time during follow-up visits. Hence, the role of the OP clinic is to optimize post-fragility fracture osteoporosis assessment by targeting patients during fracture management who would have otherwise been lost to 
TABLE 3.

Demographic information, clinical parameters, and telephone survey responses for patients in the Usual Care group ( $n=20)$

Referred to Osteoporosis Clinic

Reason patient not seen at Osteoporosis Clinic

Family physician follow-up post-fracture

BMD testing post-fragility fracture

Bisphosphonate/RANKL inhibitor post-fragility fracture Bisphosphonate

RANKL inhibitor

\author{
No referral opportunity given: 8 \\ Primary care provider managing osteoporosis care: 2 \\ Patient did not feel a need: 2 \\ Distance of clinic: 1 \\ Orthopaedic surgeon recommended against: 1 \\ Transferred from another hospital: 1 \\ More comfortable with primary care provider: 1 \\ Treatment at another facility: 1
}

19

10

6

$4 / 6$

2.6 follow up. Moreover, there is evidence that specialist OP care is cost effective compared to primary care-led interventions, as supported by an Australian study that demonstrated an incremental cost-effectiveness ratio versus standard care of $\$ 17,291$ per quality-adjusted life year gained. ${ }^{(8)}$ Proper and prompt referral of patients to specialist care is another area of needed improvement, as $8 / 20$ patients in the usual care group were not referred to the OP clinic, even though they met eligibility criteria of sustaining a fragility fracture. In addition, the mean wait time to be seen by a specialist physician was 63.7 days, which is within Osteoporosis Canada's recommended target (Tables 2 and 3). Therefore, for better access to care, there is a prominent role for improved primary care-led initial investigation and management of osteoporosis post-fragility fracture.

The current referral process of OP clinic referral consists of a nurse coordinator reviewing charts from the fracture clinic to determine whether the patient has sustained a fragility fracture. As such, this process may be prone to errors, with some patients who are not promptly and appropriately referred to specialty care. This is an area of importance, as more than half of subsequent osteoporotic fractures occur within a year of the first fracture. ${ }^{(9)}$ In terms of compliance rates with osteoporosis medications, the three-month compliance rate post-treatment initiation of $75 \%$ for the OP clinic patients reported in this study is comparable to rates cited in the literature. ${ }^{(10)}$

\section{CONCLUSION}

This is the first study highlighting the efficacy of an exclusively outpatient community FLS-type intervention in improving the use of osteoporosis medications in an outpatient community hospital setting. ${ }^{(11)}$ This is of importance, as the majority of current literature surrounding secondary fracture prevention via targeting osteoporosis risk factors has been studied in tertiary care settings, and there is a growing trend towards community-based medicine in Canada. ${ }^{(12)}$

This study was limited by the fact that it is a small, retrospective chart review and telephone survey; therefore, a cause-and-effect conclusion could not be made with regard to the impact of a hospital-based outpatient OP clinic on secondary fracture prevention, especially as it pertains to the reasons for the care-gap in OP treatment initiation. Another limitation is that patients who declined OP clinic evaluation were included in the usual care group for analysis. This may have consequently caused a bias in favour of the OP clinic group, as those who declined OP clinic evaluation may have been more likely to decline OP treatment as well. In addition, due to the retrospective design of this study, certain clinical parameters were not collected for analysis, such as time to OP treatment, which has relevance with regard to quality of care benchmarks set up by Osteoporosis Canada. Moreover, we could not delineate reasons why pharmacotherapy was refused by patients due to lack of documentation in the community, including whether there were contraindications for OP treatment.

Furthermore, pre-printed order sets have been shown to be effective in other medical sub-specialties in ensuring timely and accurate referral. ${ }^{(13)}$ Thus, future directions include OP clinic referrals in inpatient order sets to facilitate referrals to secondary fracture prevention care. In addition, educational endeavours to engage family physicians in OP management for patients with fragility fractures should be encouraged, as recent literature suggests that primary care-based management is equally effective in providing secondary fracture prevention. ${ }^{(14)}$ Subsequent larger studies involving cost analysis, morbidity, and mortality data with longer term prospective data are needed.

\section{CONFLICT OF INTEREST DISCLOSURES}

The authors declare that no conflicts of interest exist. 


\section{REFERENCES}

1. Van den Bergh JP, van Geel TA, Geusens PP. Osteoporosis, frailty and fracture: implications for case finding and therapy. Nat Rev Rheumatol. 2012;8(3):163-72.

2. McLellan AR, Gallacher SJ, Fraser M, et al. The fracture liaison service: success of a program for the evaluation and management of patients with osteoporotic fracture. Osteoporosis Int. 2003;14(12):1028-34.

3. Curtis J, Silverman SL. Commentary: The five Ws of a fracture liaison service: why, who, what, where and how? In osteoporosis, we reap what we sow. Curr Osteoporosis Rep. 2013;11(4):365-68.

4. Aizer J, Bolster MB. Fracture liaison services: promoting enhanced bone health care. Curr Rheumatol Rep. 2014;16(11):455.

5. Von Friesendorff M, McGuigan FE, Wizert A, et al. Hip fracture, mortality risk, and cause of death over two decades. Osteoporosis Int. 2016;27(10):2945-53.

6. Papaioannou A, Morin S, Cheung AM, et al. 2010 clinical practice guidelines for the diagnosis and management of osteoporosis in Canada: summary. CMAJ. 2010;182(17):1864-73.

7. Mendis AS, Ganda K, Seibel MJ. Barriers to secondary fracture prevention in primary care. Osteoporosis Int. 2017;28(10):2913-19.

8. Cooper MS, Palmer AJ, Seibel MJ. Cost-effectiveness of the Concord Minimal Trauma Fracture Liaison service, a prospective, controlled fracture prevention study. Osteoporosis Int. 2012;23(1):97-107.
9. Scaglione M, Fabbri L, Di Rollo F, et al. The second hip fracture in osteoporotic patients: not only an orthopaedic matter. Clin Cases Mineral Bone Metab. 2010;10(2):124-28.

10. Kothawala P, Badamgarav E, Ryu S, et al. Systematic review and meta-analysis of real-world adherence to drug therapy for osteoporosis. Mayo Clin Proc. 2007;82(1):1493-501.

11. Cosman F, Nicpon K, Nieves JW. Results of a fracture liaison service on hip fracture patients in an open healthcare system. Aging Clin Exp Res. 2017;29(2):331-34.

12. $\mathrm{Wu} \mathrm{CH}$, Chen $\mathrm{CH}$, Chen $\mathrm{PH}$, et al. Identifying characteristics of an effective fracture liaison service: systematic literature review. Osteoporosis Int. 2018;29(5):1023-47.

13. Doyle MA, Brez S, Sicoli S, et al. Using standardized insulin orders to improve patient safety in a tertiary care centre. Can J Diabetes. 2014;38(2):118-25.

14. Ganda K, Schaffer A, Pearson S, et al. Compliance and persistence to oral bisphosphonate therapy following initiation within a secondary fracture prevention program: a randomised controlled trial of specialist vs. non-specialist management. Osteoporosis Int. 2014;25(4):1345-55.

Correspondence to: Timothy S.H. Kwok, Department of Medicine, University of Toronto, 200 Elizabeth St., Eaton Building 14-217, Toronto, ON M5G 2C4, Canada

E-mail: timothysh.kwok@mail.utoronto.ca 